\title{
Cerebellar plasticity and associative memories are controlled by perineuronal nets
}

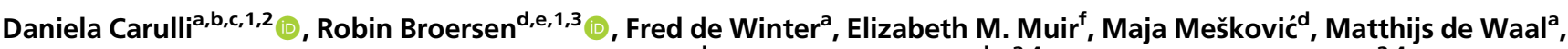 \\ Sharon de Vries ${ }^{a}$, Henk-Jan Boele ${ }^{\text {, }}$ Cathrin B. Canto ${ }^{d, e}$, Chris I. De Zeeuw ${ }^{d, e, 2,4}$, and Joost Verhaagen ${ }^{a, g, 2,4}$
}

\begin{abstract}
aLaboratory for Neuroregeneration, Netherlands Institute for Neuroscience, Royal Academy of Arts and Sciences, 1105 BA Amsterdam, The Netherlands: ${ }^{b}$ Department of Neuroscience Rita Levi-Montalcini, University of Turin, 10040 Turin, Italy; ' Neuroscience Institute Cavalieri Ottolenghi, University of Turin, 10040 Turin, Italy; dDepartment of Cerebellar Coordination and Cognition, Netherlands Institute for Neuroscience, Royal Academy of Arts and Sciences, 1105 BA Amsterdam, The Netherlands; 'Department of Neuroscience, Erasmus Medical Center (MC), 3015 CN Rotterdam, The Netherlands; ${ }^{\circ}$ Department of Physiology, Development and Neuroscience, University of Cambridge, Cambridge CB2 3EG, United Kingdom; and ${ }^{9}$ Department of Molecular and Cellular Neurobiology, Center for Neurogenomics and Cognition Research, Vrije Universiteit Amsterdam, 1081 HV Amsterdam, The Netherlands
\end{abstract}

Edited by Peter L. Strick, University of Pittsburgh, Pittsburgh, PA, and approved February 10, 2020 (received for review September 18, 2019)

Perineuronal nets (PNNs) are assemblies of extracellular matrix molecules, which surround the cell body and dendrites of many types of neuron and regulate neural plasticity. PNNs are prominently expressed around neurons of the deep cerebellar nuclei (DCN), but their role in adult cerebellar plasticity and behavior is far from clear. Here we show that PNNs in the mouse DCN are diminished during eyeblink conditioning (EBC), a form of associative motor learning that depends on DCN plasticity. When memories are fully acquired, PNNs are restored. Enzymatic digestion of PNNs in the DCN improves EBC learning, but intact PNNs are necessary for memory retention. At the structural level, PNN removal induces significant synaptic rearrangements in vivo, resulting in increased inhibition of DCN baseline activity in awake behaving mice. Together, these results demonstrate that PNNs are critical players in the regulation of cerebellar circuitry and function.

cerebellum | plasticity | perineuronal net | eyeblink conditioning | learning

$\mathbf{P}$ erineuronal nets (PNNs) are lattice-like aggregates of extracellular matrix molecules surrounding the cell body and dendrites of various types of neurons in the central nervous system (CNS). Synaptic contacts impinging on PNN-bearing neurons are embedded in these structures. Therefore, PNNs are strategically positioned to influence the development and stabilization of synaptic connections. PNNs emerge during late postnatal development, in coincidence with the closure of critical periods for experiencedependent plasticity (1). Accumulating evidence suggests that PNNs inhibit different forms of CNS plasticity in adult life, under both physiological and pathological conditions. For instance, enzymatic digestion of PNNs with chondroitinase $\mathrm{ABC}$ (ch'ase) or manipulation of PNN components enhance cortical plasticity (2-7), cognitive flexibility (8), AMPA receptor-dependent synaptic plasticity (9), and axonal sprouting after injury $(3,10)$. PNN disruption also increases the formation of recognition memory (11), whereas it impairs the consolidation of other types of memory (12-17).

PNNs are composed of a meshwork of interconnected proteins and carbohydrates. Hyaluronan represents the backbone of the PNN structure, to which chondroitin sulfate proteoglycans (CSPGs) are bound. The binding between hyaluronan and CSPGs is stabilized by link proteins $(1,3)$. CSPGs consist of a core protein and a variable number of chondroitin sulfate side chains, which are the target of the enzyme ch'ase. The sulfation pattern of chondroitin sulfates is critical for PNN function as it encodes specific information for the binding of plasticity regulators (18-20).

The cerebellum shows abundant PNNs around neurons in the deep cerebellar nuclei (DCN) (21). The cerebellum plays a pivotal role in motor, emotional and cognitive associative learning (22). Delay eyeblink conditioning (EBC) is an associative learning paradigm, which consists of an eyelid closure in response to an initially neutral stimulus (such as a light; conditioned stimulus [CS]) after repeated pairing of the CS with an obligatory eyeblinkeliciting stimulus (such as an air puff; unconditioned stimulus [US]). EBC critically depends on DCN function (23). CS and US inputs reach the DCN via excitatory collaterals of mossy fibers originating in the basilar pontine nuclei and of climbing fibers originating in the inferior olive, respectively (24). While mossy fibers can modulate Purkinje cells in the cerebellar cortex through their innervation of the granule cells that give rise to the parallel fibers, the climbing fibers excite the Purkinje cells directly. Purkinje cells in turn inhibit the DCN neurons, allowing an integration of the excitatory collaterals with inhibitory inputs (25). At the end of EBC the activity of DCN neurons is enhanced during expression of the conditioned response (CR) (26). Despite the presence of PNNs, multiple forms of synaptic and structural plasticity take place in the DCN during EBC. For example, sprouting of mossy fiber collaterals is observed in the DCN after EBC, and the number of new mossy fiber varicosities in the DCN areas implicated in the EBC is positively correlated with the amplitude of CR (27). This evidence raises the possibility that structural

\section{Significance}

Understanding mechanisms underlying learning and memory is crucial in view of tackling cognitive decline occurring during aging or following neurological disorders. The cerebellum offers an ideal system to achieve this goal because of the wellcharacterized forms of motor learning that it controls. It is so far unclear whether cerebellar memory processes depend on changes in perineuronal nets (PNNs). PNNs are assemblies of extracellular matrix molecules around neurons, which regulate neural plasticity. Here we demonstrate that during eyeblink conditioning (EBC), which is a form of cerebellar motor learning, PNNs in the mouse deep cerebellar nuclei are dynamically modulated, and PNN changes are essential for the formation and storage of EBC memories. Together, these results unveil an important mechanism controlling motor associative memories.

Author contributions: D.C., R.B., C.B.C., C.I.D.Z., and J.V. designed research; D.C., R.B., F.d. W., M.M., M.d.W., and S.d.V. performed research; E.M.M. and H.-J.B. contributed new reagents/analytic tools; D.C. and R.B. analyzed data; and D.C., R.B., C.I.D.Z., and J.V. wrote the paper.

The authors declare no competing interest.

This article is a PNAS Direct Submission.

This open access article is distributed under Creative Commons Attribution-NonCommercialNoDerivatives License 4.0 (CC BY-NC-ND).

Data deposition: Datasets and analyses have been deposited in the online open access repository Figshare and can be accessed via https://figshare.com/collections/Cerebellar plasticity_and_associative_memories_are_controlled_by_perineuronal_nets/4814616/1.

${ }^{1}$ D.C. and R.B. contributed equally to this work.

${ }^{2}$ To whom correspondence may be addressed. Email: d.carulli@nin.knaw.nl, c.de.zeeuw@ nin.knaw.nl, or j.verhaagen@nin.knaw.nl.

${ }^{3}$ Present address: Eccles Institute of Neuroscience, The John Curtin School of Medical Research, Australian National University, Canberra, ACT 2601, Australia.

${ }^{4}$ C.I.D.Z. and J.V. contributed equally to this work

This article contains supporting information online at https://www.pnas.org/lookup/suppl/ doi:10.1073/pnas.1916163117/-/DCSupplemental.

First published March 9, 2020. 
plasticity in the DCN may be induced or facilitated by a decrease in PNNs.

In the current study, we sought to unravel the role of PNNs in the control of cerebellar plasticity at the circuit and behavioral level. We investigated whether expression of PNN-CSPGs in the $\mathrm{DCN}$ is altered during acquisition and consolidation of $\mathrm{EBC}$ in adult mice. Moreover, we overexpressed ch'ase in the $\mathrm{DCN}$ via a lentiviral vector and assessed whether PNN-CSPG digestion impacts the performance of mice both during and after formation of EBC memories. Finally, to establish a link between behavioral changes and circuit plasticity, we examined the effects of PNN digestion on remodeling of inhibitory and excitatory synaptic terminals and on the baseline electrophysiological properties of DCN neurons in vivo. Our data provide evidence for a dynamic modulation of PNNs in response to EBC and for a tight control of the balance between excitatory and inhibitory inputs to DCN neurons by PNNs, thereby regulating acquisition and retention of EBC memory.

\section{Results}

PNNs Are Dynamically Regulated During EBC. To investigate the role of PNNs in cerebellum-dependent associative learning, we first examined the expression of PNNs enwrapping cerebellar nuclei neurons over the course of EBC. We hypothesized that PNNs are modulated in response to EBC. We examined PNNs during two phases, namely, during learning (day 5) and when animals had reached stable levels of performance (day 10) $(28,29)$. PNN expression was evaluated by quantifying the staining intensity of Wisteria floribunda agglutinin (WFA; a general marker for PNN chondroitin sulfates (30)) around individual neurons in eyeblinkencoding regions of the DCN, i.e., the dorsolateral hump (DLH) of the anterior interpositus nucleus (IntA) and the adjacent lateral part of the IntA $(26,28,31)$, with the lateral nucleus as control area (Fig. $1 A$ and $B$ ). We found a significant increase in CRs (from $1 \%$ on day 1 to $60 \%$ on day 5 ) over the course of 5 days (d) training in the conditioned group, whereas, as expected, pseudoconditioned animals did not show learning (day, $F(1.971$,
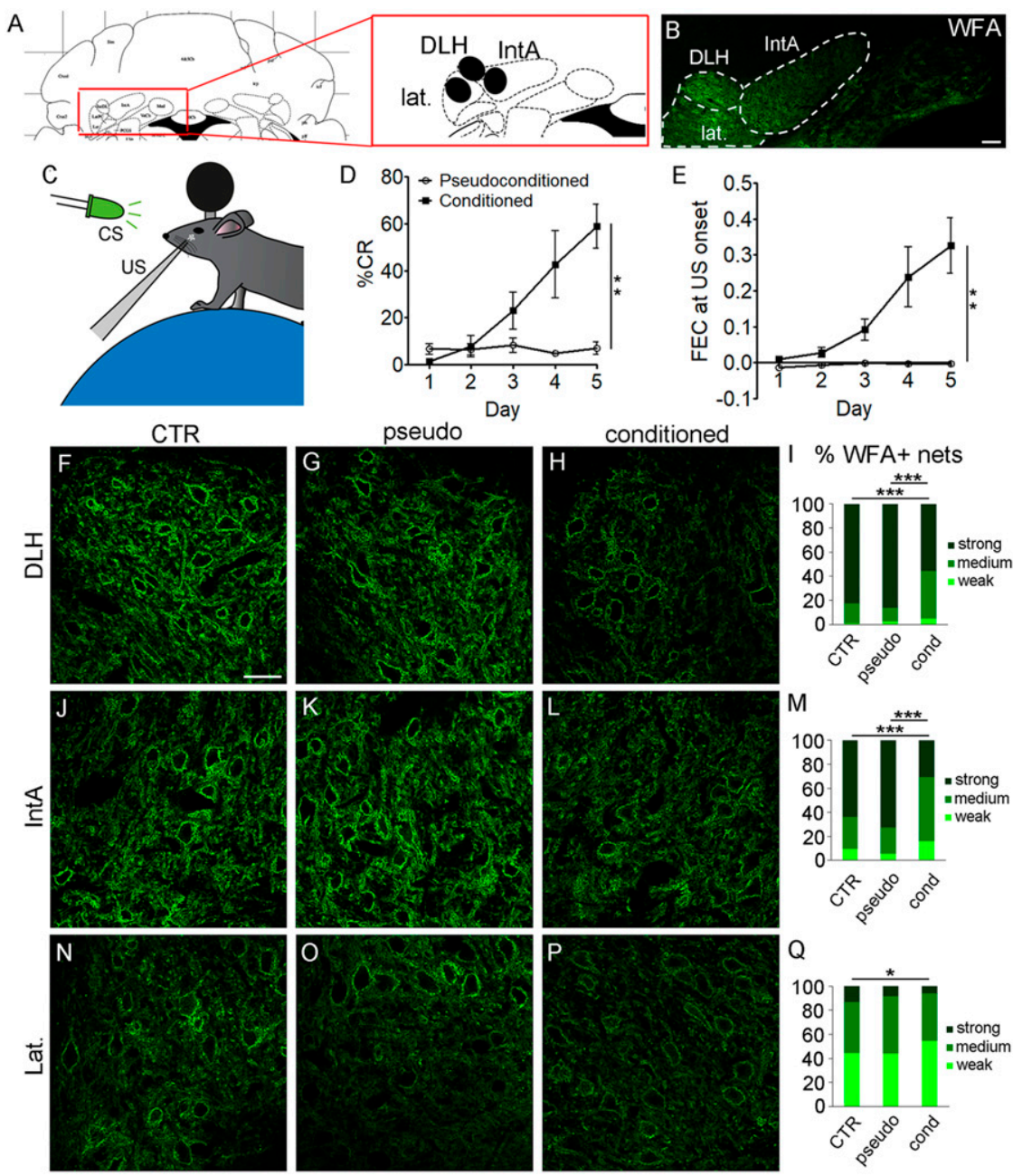

Fig. 1. PNNs are reduced during acquisition of EBC. (A) Scheme of a coronal section of the cerebellum [bregma: $-6.12 \mathrm{~mm}$, from Paxinos and Franklin (81)], showing the location in which the analysis of WFA staining intensity has been performed (black circles). (B) WFA staining in the DCN, where the analyzed nuclei (lat., lateral nucleus) are outlined by dashed lines. $(C)$ Mice were head-fixed on a cylindrical treadmill during EBC training sessions. ( $D$ and $E$ ) Mice were subjected to EBC (conditioned) or unpaired presentation of CS and US (pseudoconditioned). Learning was observed in the conditioned group as an increase in percentage of trials with CRs $(D)$ and increase in FEC at the time of US onset $(E)$. ( $F-Q)$ The intensity of WFA+ nets was analyzed in the DLH, the lateral part of the IntA, and the lateral nucleus in CTR mice, in mice that were trained for $5 \mathrm{~d}$ (conditioned mice), and in pseudoconditioned (pseudo) mice. A significant shift toward weak and medium intensity nets is apparent in conditioned mice when compared to CTR and pseudo mice in the DLH ( $F-I)$ and the IntA $(J-M)$. See also $S I$ Appendix, Fig. S1. In the lateral nucleus, there is only a slight decrease in WFA intensity in conditioned mice when compared to CTR mice ( $N-Q)$. There is no difference in the frequency distribution of WFA+ nets between CTR and pseudo animals in all nuclei $(I, M$, and $Q) .{ }^{*} P<0.05, * * P<0.01, * \star \star P<0.001$. (Scale bars, $100 \mu \mathrm{m}$ in $B$ and $50 \mu \mathrm{m}$ in $F$ [also applies to $G, H, J-L$, and $N-P$ ].) 
$15.767)=13.334, P<0.001$; group, $F(1,8)=17.332, P=0.003$; interaction: $F(1.971,15.767)=13.337, P<0.001$; GreenhouseGeisser corrected repeated-measures [RM] ANOVA; Fig. 1D). Conditioned mice also displayed an increase in the fraction eyelid closure (FEC) at US onset, in contrast to pseudoconditioned mice $($ day, $F(1.746,13.965)=11.229, P=0.002$; group, $F(1,8)=14.523$, $P=0.005$; interaction, $F(1.746,13.965)=10.157, P=0.002$; Greenhouse-Geisser corrected RM ANOVA; Fig. 1E). Since neurons of the DCN display natural variability in WFA staining intensity, we divided the PNNs in three categories: strong, medium, and weak nets (as in ref. 32). Data from both ipsilateral and contralateral cerebellar hemispheres (relative to US) were analyzed because both sides encode EBC (33) and axonal plasticity has been reported for both sides (27). WFA intensity distributions were not different between the right and left side in any of the three subnuclei (DLH, IntA, and lateral) in control (CTR; i.e., mice which did not receive stimuli) and in pseudoconditioned mice (DLH, CTR, $\mathrm{X}^{2}{ }_{2}=0.79, P=0.67$; pseudo, $\mathrm{X}^{2}{ }_{2}=2.03, P=$ 0.36 ; IntA, CTR, $\mathrm{X}_{2}^{2}=3.82, P=0.15$; pseudo, $\mathrm{X}_{2}^{2}=0.52, P=0.77$; lateral nucleus, CTR, $\mathrm{X}_{2}^{2}=5.28, P=0.07$; pseudo, $\mathrm{X}_{2}{ }_{2}=1.74, P=$ 0.42 ). Data from the two sides were therefore pooled together. Moreover, no difference was found between pseudoconditioned and control mice after $5 \mathrm{~d}$ of EBC (CTR DLH versus pseudo DLH, $\mathrm{X}^{2}{ }_{2}=4.24, P=0.12$; CTR IntA versus pseudo IntA, $\mathrm{X}_{2}^{2}=3.13, P=$ 0.21 ; CTR lateral versus pseudo lateral, $\mathrm{X}_{2}^{2}=2.48, P=0.29$; Fig. 1 $F, G, I-K, M-O$, and $Q)$, indicating that unpaired presentation of CS and US does not affect PNN expression in the DCN. Interestingly, both in the IntA and the DLH of $5 \mathrm{~d}$ conditioned mice, the percentage of strong nets significantly decreased on both sides when compared to pseudoconditioned and control mice (DLH, from 85 to $\sim 55 \%$; IntA, from $\sim 65$ to $\sim 30 \%$ ), whereas the percentage of medium and weak nets increased (medium nets, DLH, from $\sim 15$ to $\sim 40 \%$; IntA, from $\sim 25$ to $\sim 50 \%$; weak nets, DLH, from $\sim 1$ to $\sim 5 \%$; IntA, from $\sim 8$ to $\sim 16 \%$; CTR DLH versus conditioned DLH, $\mathrm{X}_{2}^{2}=31.97, P<0.001$; CTR IntA versus conditioned IntA, $\mathrm{X}^{2}{ }_{2}=39.30, P<0.001$; pseudo DLH versus conditioned DLH, $\mathrm{X}_{2}^{2}=40.90, P<0.001$; pseudo IntA versus conditioned IntA, $\mathrm{X}_{2}{ }_{2}=$ 51.95, $P<0.001$; Fig. $1 F-M)$. In addition, in the DLH of conditioned mice, the decrease in WFA intensity was more pronounced on the right side than on the left side $\left(\mathrm{X}^{2}{ }_{2}=6.59, P=0.04 ; S I\right.$ Appendix, Fig. S1A). No difference between right and left side was detected in the IntA of conditioned mice $\left(\mathrm{X}^{2}{ }_{2}=3.75, P=0.15 ; S I\right.$ Appendix, Fig. S1B). The effect of EBC on PNN expression in the lateral nucleus was substantially less remarkable. The percentage of strong nets decreased only by $\sim 5 \%$ and was statistically significant only when comparing control and conditioned mice $\left(\mathrm{X}_{2}^{2}=7.03\right.$, $P<0.05$; pseudo versus conditioned mice, $\mathrm{X}_{2}^{2}=3.76, P=0.15$; Fig. $1 N-Q)$.

To find out whether the decrease in WFA intensity in conditioned animals may be linked directly to the learning process, we also analyzed WFA intensity in the DLH and IntA of animals that showed poor learning rate after $5 \mathrm{~d}$ of $\mathrm{EBC}(\% \mathrm{CR},<35 \%$; $n=6)$. Interestingly, in those animals, the frequency distribution of WFA+ nets in the DLH was not different from that of CTR mice $\left(\mathrm{X}_{2}{ }_{2}=0.11, P=0.95\right.$; SI Appendix, Fig. S1C). In the IntA of poor learners, the percentage of strong nets was higher than in good learners (poor learners, 50\%; good learners, $\sim 30 \% ; \mathrm{X}_{2}^{2}=$ 16.40, $P<0.001$ ), although slightly lower than in CTR mice (CTR, $\sim 65 \% ; \mathrm{X}_{2}^{2}=17.93, P<0.001 ;$ SI Appendix, Fig. S1D). Furthermore, taking into consideration both good and poor learners, the amount of strong nets in the DLH showed a significant inverse correlation with the \% of CR (Pearson's correlation $=-0.63, P<0.05 ;$ SI Appendix, Fig. S1E). No significant correlation between $\%$ of strong nets and $\%$ of CR could be established in the IntA (Pearson's correlation $=0.27, P=0.42 ; S I$ Appendix, Fig. S1F). These data strengthen the hypothesis that it is the PNN reduction in the DLH that contributes to EBC acquisition.
We also evaluated whether the number of neurons in the DLH or IntA that were surrounded by WFA+ PNNs was changed after $5 \mathrm{~d}$ of EBC or pseudoconditioning. No difference in the percentages of WFA-enwrapped neurons was found among conditioned, pseudoconditioned, or control mice (DLH, CTR, 88.94\% $\pm 1.85 \%$; pseudo, $85.56 \% \pm 2.24 \%$; cond., $89.27 \% \pm 2.05 \%$; one-way ANOVA $F(2,13)=1.067, P=0.37$; IntA, CTR, $91.46 \% \pm 1.69 \%$; pseudo, $87.44 \% \pm 1.04 \%$; cond., $92.91 \% \pm 1.03 \%$; one-way ANOVA $F(2,13)=3.378, P=0.07$; SI Appendix, Fig. S1 $G$ and $H)$. This suggests that EBC does not alter the number of neurons bearing a PNN but modulates the amount of chondroitin sulfates contained in PNNs.

In order to ascertain whether EBC also affects protein constituents of the PNN, we evaluated the staining intensity of the CSPG aggrecan, which is crucial for PNN integrity (6), in mice subjected to EBC for $5 \mathrm{~d}$. The vast majority of WFA+ nets in the DCN contained aggrecan (see also ref. 21). We found a significant decrease in aggrecan staining in the DLH of conditioned mice when compared to CTR and pseudoconditioned mice (weak nets, $50 \%$ in CTR and pseudomice, $70 \%$ in EBC-mice; strong nets, $10 \%$ in CTR and pseudomice, $0 \%$ in EBC-mice; CTR vs. pseudo, $\mathrm{X}_{2}{ }_{2}=0.32, P=0.85$; CTR vs. conditioned, $\mathrm{X}^{2}{ }_{2}=16.07, P<0.001$; pseudo vs. conditioned, $\mathrm{X}^{2}{ }_{2}=18.00, P<$ 0.001; SI Appendix, Fig. S2 $A-D$ ). A similar, albeit smaller, decrease in aggrecan staining was found in the IntA (weak nets, $\sim 25 \%$ in CTR and pseudomice, $35 \%$ in EBC-mice; strong nets, $8 \%$ in CTR and pseudomice, $0 \%$ in EBC-mice; CTR vs. pseudo, $\mathrm{X}_{2}^{2}=3.99, P=0.14$; CTR vs. conditioned, $\mathrm{X}_{2}{ }_{2}=14.40, P<$ 0.001 ; pseudo vs. conditioned, $\mathrm{X}_{2}{ }_{2}=10.06, P<0.01$; SI Appendix, Fig. S2E). The effect of EBC on aggrecan expression in the lateral nucleus was negligible $\left(\mathrm{X}_{2}{ }_{2}=5.90, P=0.20\right.$; SI Appendix, Fig. $\mathrm{S} 2 F$ ).

To assess whether PNN-CSPG expression was also altered when animals had completed the acquisition phase and had reached a plateau in learning, we trained conditioned $(n=3)$ and pseudoconditioned mice $(n=3)$ for $10 \mathrm{~d}$. Conditioned mice showed learning as an increase in CRs (from 2\% [day 1] to $75 \%$ [day 10]; day, $F(9,40.452)=13.941, P<0.001$; group, $F(1,9.798)=$ 104.638, $P<0.001$; interaction, $F(9,40.452)=11.375, P<0.001$, generalized linear mixed model (GLMM); Fig. $2 A)$ and FEC at US onset (day, $F(9,40.163)=9.338, P<0.001$; group, $F(1,15.301)=$ 50.729, $P<0.001$; interaction, $F(9,40.163)=8.514, P<0.001$, GLMM; Fig. $2 B$ ). A transient decrease in performance on day 6 to 7 was noticed, likely due to the absence of training in the two preceding days. As WFA intensity between right and left sides did not differ in either the DLH or the lateral IntA following conditioning (DLH, $\mathrm{X}_{2}{ }_{2}=1.36, P=0.51$; IntA, $\mathrm{X}_{2}^{2}=1.47, P=0.48$ ), we pooled the data from both sides. In contrast to our findings after $5 \mathrm{~d}$ of EBC, after $10 \mathrm{~d}$ the WFA intensity distribution in the IntA and the DLH was not different between conditioned and pseudoconditioned mice (DLH, $\mathrm{X}_{2}{ }_{2}=0.74, P=0.69$; IntA, $\mathrm{X}_{2}{ }_{2}=0.052$, $P=0.97$; Fig. $2 C-H$ ). These data indicate that PNNs are transiently reduced during associative motor learning and are restored when the memory trace has been acquired.

PNN Digestion Enhances Acquisition but Impairs Retention of EBC. Based on the observed modulation of PNNs during EBC, we asked whether PNNs are necessary for acquisition and/or retention of this associative memory. To address this question, we digested PNNCSPGs in the IntA and DLH with ch'ase overexpressed by means of a lentiviral vector (LV) to ensure long-term, stable expression of the enzyme (34). Control mice received injections of LV overexpressing GFP. In LV-GFP mice, GFP expression was detected in the DCN (Fig. $3 A$ ) as early as 1 wk postinjection, and this was maintained at least up to $7 \mathrm{wk}$ (longest time point analyzed). CSPG amount was assessed by WFA labeling. In LV-ch'ase mice, WFA staining in the IntA and DLH was virtually completely abolished ( $\sim 95 \%$ decrease; Fig. $3 B-D)$. Qualitative observations revealed that the extent of 

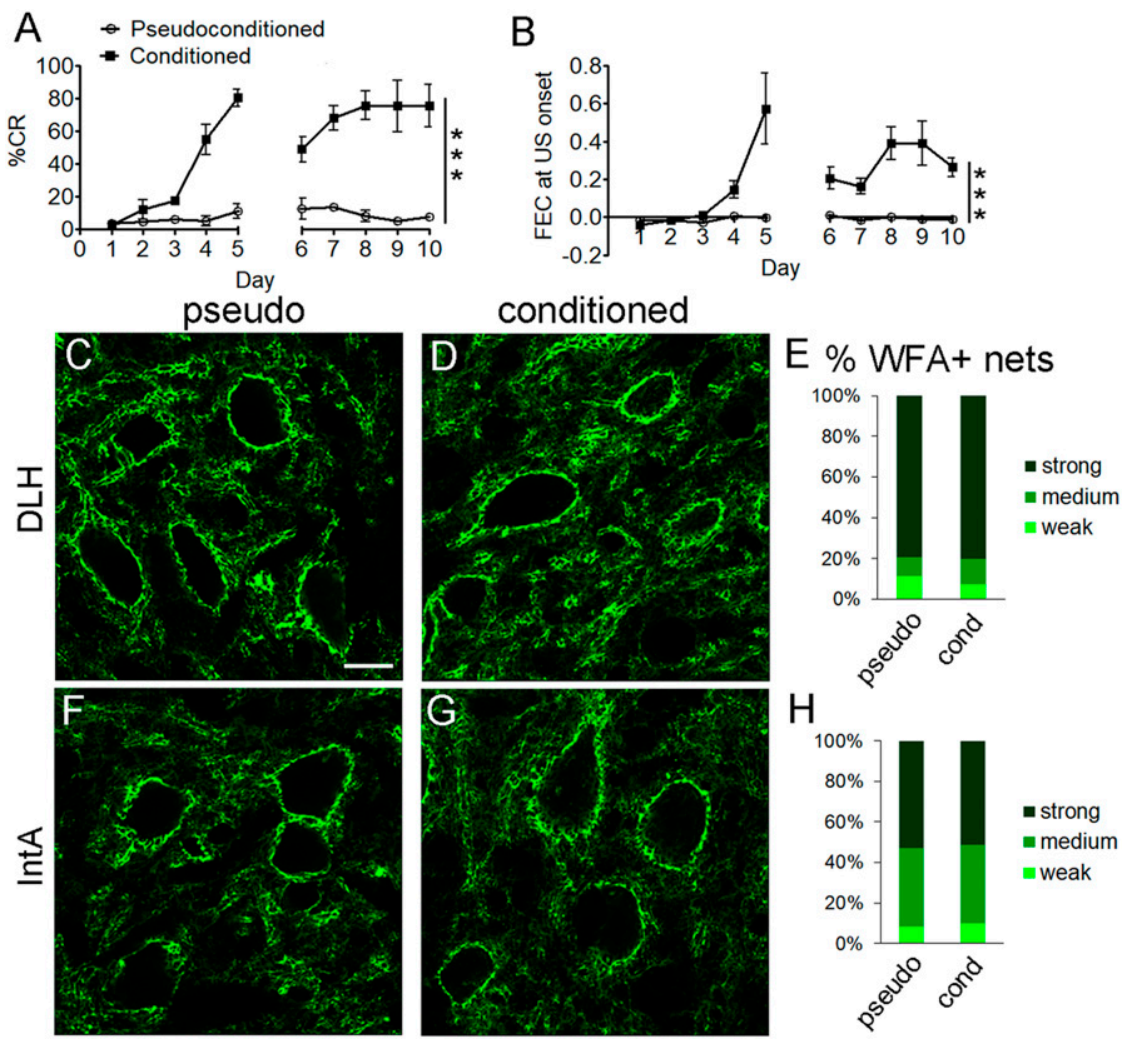

Fig. 2. PNNs are restored during consolidation of EBC memory. ( $A$ and $B$ ) Mice were subjected to 10 sessions of EBC (conditioned) or pseudoconditioned stimulation. Conditioned mice learn during $10 \mathrm{~d}$ of training, as shown by their $\% C R(A)$ and FEC at the US onset $(B)$. (C- $H$ ) WFA staining intensity of PNNs in the DLH (C-E) and the lateral IntA $(F-H)$ is not different between conditioned mice and pseudoconditioned (pseudo) mice on day 10 . The frequency distribution of weak, medium, and strong WFA+ nets in the DLH and IntA is shown in $E$ and $H$, respectively. $* * * P<0.001$. (Scale bar, $15 \mu \mathrm{m}$ in $C$ [also applies to $D$, $F$, and $G]$.)

PNN digestion was comparable in animals killed at 2, 4, and $7 \mathrm{wk}$ after LV-ch'ase injection.

We examined whether removal of PNN-CSPGs by LV-ch'ase affected EBC learning and memory (see Fig. $4 A$ for timeline of the experiment). Both LV-GFP $(n=22)$ and LV-ch'ase $(n=19)$ mice showed a significant increase in $\%$ of CRs over the course of EBC acquisition (LV-GFP, from 4 to 50\%; LV-ch'ase, from 6 to $70 \% ; F(4,39.447)=56.613, P<0.001$; Fig. $4 B)$. However, LV-ch'ase mice learned significantly faster and better (group, $F(1,41.530)=$ $6.672, P=0.013$; interaction, $F(4,39.447)=3.403, P=0.018$; Fig. $4 B)$. This effect was supported by an accelerated increase in FEC at US onset (day, $F(4,39.457)=30.832, P<0.001$; group, $F(1,41.209)=$ 4.691, $P=0.036$; interaction, $F(4,39.457)=2.074, P=0.103$, GLMM; Fig. $4 C$ ). These results indicate that ch'ase overexpression in the IntA and DLH improves cerebellum-dependent learning.
Since we observed that WFA staining intensity was restored after $10 \mathrm{~d}$ of EBC (Fig. $2 \mathrm{C}-\mathrm{H}$ ), we hypothesized that long-term removal of PNNs would disrupt EBC retention. To test this hypothesis, we compared EBC performance over a period of $21 \mathrm{~d}$, during which mice were intermittently retrained with short training sessions. Each memory retention session consisted of $25 \%$ of a full acquisition session. For this test we included only mice that had shown evidence of learning during the acquisition phase, i.e., mice reaching $>35 \%$ CR trials on day 5 of acquisition (LV-GFP, $n=16$; LV-ch'ase, $n=$ 18). Performance metrics of these mice on acquisition day 5 were comparable (LV-GFP versus LV-ch'ase, \% CR, $64.2 \pm 18.6 \%$ versus $71.4 \pm 18.9 \%, t(32)=-1.124, P=0.27$; FEC at US onset, $0.42 \pm$ 0.22 versus $0.42 \pm 0.18, t(32)=-0.075, P=0.941)$. We found that over the course of the memory retention phase, the performance of LV-GFP mice decreased in the first week and then remained
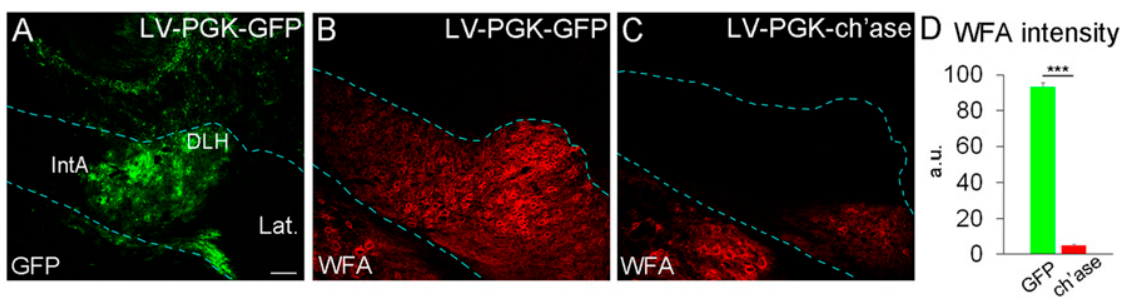

Fig. 3. Transduction efficiency of LV-PGK-GFP and LV-PGK-ch'ase in the DCN. (A) Four weeks after LV-GFP injection, GFP is strongly overexpressed in the DCN and the cerebellar cortex (dorsal to the DCN). ( $B$ and $C$ ) PNNs, detected by WFA, are dramatically reduced following LV-ch'ase injection in the DCN ( $C$ ), when compared to DCN injected with LV-GFP $(B)$. $D$ shows the quantification of WFA intensity in the IntA of GFP- and ch'ase-injected mice. In $A-C$, the DCN are outlined by dashed lines. a.u., arbitrary units. $* * * P<0.001$. (Scale bar, $100 \mu \mathrm{m}$ in $A$ [also applies to $B$ and $C$ ].) 
A
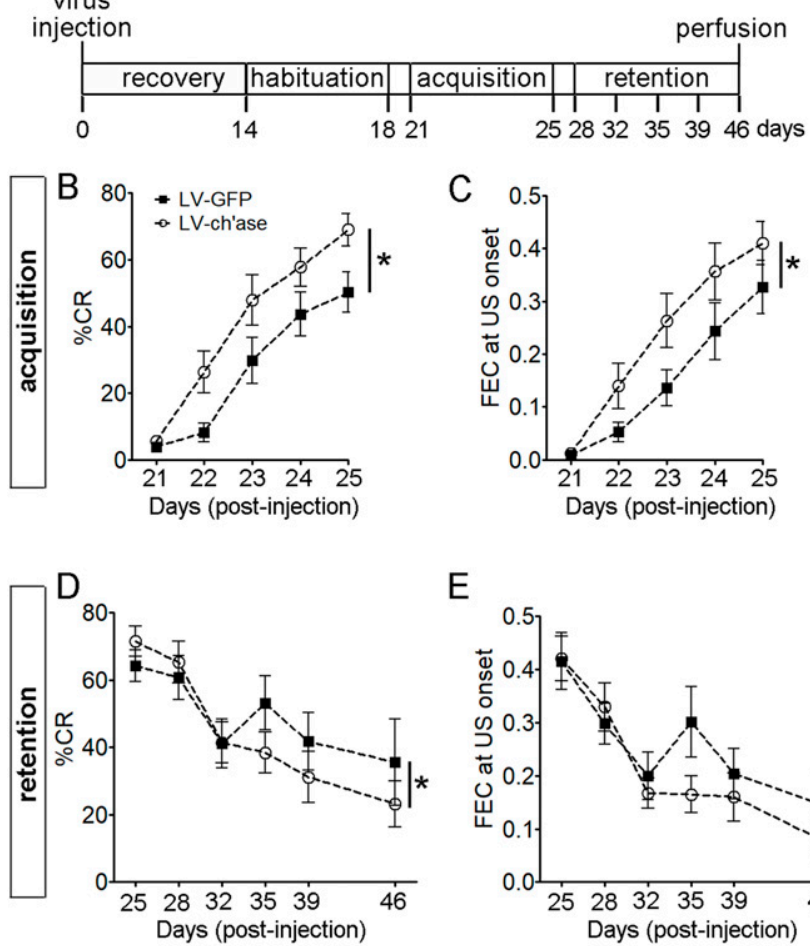

E
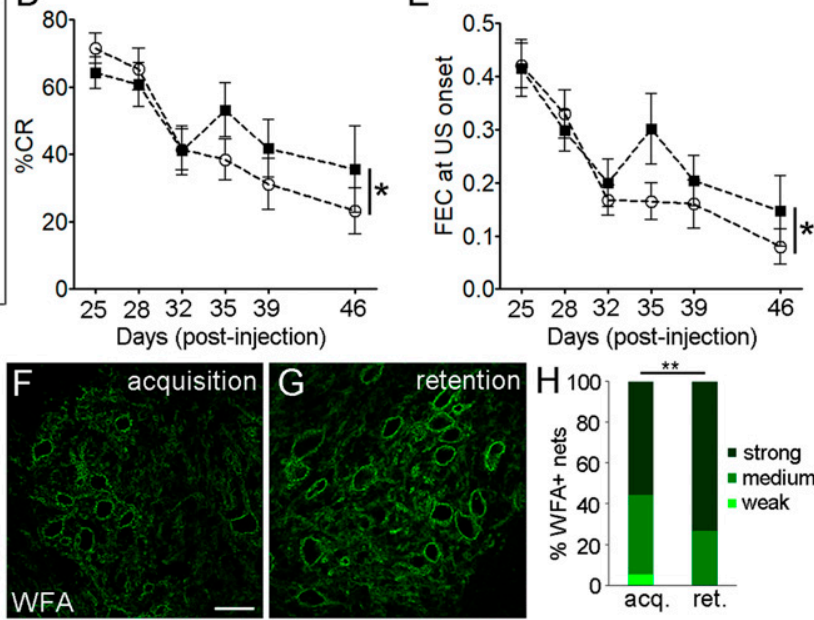

Fig. 4. Effect of PNN digestion on EBC acquisition and retention. (A) Timeline of the experimental design. $(B-E)$ EBC performance was calculated as \%CR and FEC at US onset and was compared between LV-GFP and LVch'ase mice during memory acquisition $(B$ and $C$ ) and memory retention ( $D$ and $E$ ). During memory acquisition we found an increase in \% $\mathrm{CR}(B)$ and FEC at US onset (C) over $5 \mathrm{~d}$ in both groups, as well as a significant difference between groups and an interaction effect, with ch'ase mice showing better performance. ( $D$ and $E$ ) For testing memory retention we included only mice that had shown clear evidence of learning during the acquisition phase, i.e., mice reaching $>35 \%$ CR trials on day 5 of acquisition. Performance metrics of those LV-GFP and LV-ch'ase mice on acquisition day 5 were comparable (see day 25 postinjection in $D$ and $E$ ). During memory retention, ch'ase mice show worse performance, with \%CR significantly different in the interaction days $\times$ groups $(D)$ and FEC at US onset significantly different between groups from retention day 10 onward $(E)$. $(F-H)$ The amount of PNNs with strong WFA intensity in the DLH of LV-GFP mice tested for memory retention is higher than in mice tested for memory acquisition (after $5 \mathrm{~d}$ of EBC). ${ }^{*} P<$ $0.05, * * P<0.01$. (Scale bar, $50 \mu \mathrm{m}$ in $F$ [also applies to $G$ ].)

relatively stable, whereas LV-ch'ase mice exhibited a continuous decline with time, as shown by their \% of CR trials (day, $F(4,22.384)=11.533, P<0.001$; group, $F(1,30.703)=0.986, P=$ 0.329 ; interaction, $F(4,22.384)=3.185, P=0.033$, GLMM; Fig. $4 D$ ) and FEC at US onset (D10 to D21, day, $F(2,23.956)=2.968$; $P=0.071$; group, $F(1,30.506)=4.339, P=0.046$; interaction, $F(2,23.956)=0.84, P=0.444$, GLMM; Fig. $4 E)$. Interestingly, PNNs in the DLH of LV-GFP mice at the end of the retention phase exhibit stronger WFA staining intensity than at the end of the acquisition phase $\left(\mathrm{X}^{2}{ }_{2}=10.99, P<0.01\right.$; Fig. $\left.4 F-H\right)$. Together, these data support the hypothesis that retention of the EBC memory trace requires a well-developed PNN.
Digestion of PNNs Leads to an Increased Number of GABAergic Terminals in the DCN. To pinpoint the neural substrate of the altered learning and memory capacities of mice after PNN digestion, we examined whether ch'ase affects the organization of DCN circuitry in vivo, focusing on morphological changes of GABAergic and glutamatergic axon terminals in the IntA, including the DLH. The vast majority of synaptic terminals in the DCN are GABAergic and belong to Purkinje cells, as revealed by double staining for VGAT (which is contained in GABAergic terminals) and calbindin (which is expressed by Purkinje cells; $S I$ Appendix, Fig. S3 $A$ and $B$, and ref. 35). GABAergic terminals are particularly abundant around the soma of DCN neurons (SI Appendix, Fig. S3A). PNNs in the DCN surround non-GABAergic (putatively glutamatergic) neurons $(21,36)$, which are characterized by a larger cell body size than inhibitory neurons (37). Therefore, the effect of ch'ase on the morphology of GABAergic terminals was evaluated around the soma of large neurons (cell body size $>250 \mu \mathrm{m}^{2}$ ). We found that in ch'ase-treated cerebella, GABAergic terminals appeared to be less distinct, forming a more continuous layer around the neuronal soma than in control cerebella (Fig. $5 A-H$ ). Indeed, the number of VGAT-negative spaces along the neuronal soma in ch'ase-injected IntA was strongly decreased when compared to the uninjected side or GFP-injected IntA (uninjected, $185.44 \pm 7.20$ troughs in VGAT intensity profile per mm neuronal membrane; $n=34$ neurons; GFP, $161.29 \pm$ 6.24; $n=52$ neurons; ch'ase, $86.24 \pm 7.33, n=33$ neurons; one-way ANOVA $F(2,116)=42.24 ; P<0.001$; Tukey's post hoc test, uninjected or GFP versus ch'ase, $P<0.001$; SI Appendix, Fig. S4 $A-$ $D)$. No difference between GFP-injected and uninjected IntA was detected (Tukey's post hoc test, $P>0.05 ;$ SI Appendix, Fig. S4 A, B, and $D)$.

To study the ultrastructure of the GABAergic synapses following ch'ase treatment, we performed electron microscopy. GABAergic boutons were identified by the presence of oval synaptic vesicles and symmetric release sites. Glutamatergic terminals were identified by the presence of rounded synaptic vesicles and thick postsynaptic densities $(38,39)$ (SI Appendix, Fig. S5 $A$ and $B^{\prime}$ ). In accordance with the immunohistochemical data, GABAergic boutons were abundant around the cell body of DCN neurons (Fig. 5I). After ch'ase treatment, GABAergic boutons did not display gross morphological abnormalities, showing clear synaptic vesicles, mitochondria, and release sites (Fig. 5K). However, ch'ase induced a substantial increase in the number of GABA+ terminals (uninjected, $438.62 \pm 17.75$ terminals per mm; ch'ase, $553.47 \pm 18.27$ terminals per mm; Student's $t$ test $\mathrm{t}_{40}=4.23, P<0.001$; Fig. $\left.5 \mathrm{~J}-L\right)$. In some cases, in ch'ase-treated cerebella, inhibitory terminals appeared squeezed along the neuronal membrane (Fig. $5 K$ ). Indeed, the average distance between GABAergic terminals was much lower than in control DCN (uninjected, $183.05 \pm 27.64 \mathrm{~nm}$; ch'ase, $97.44 \pm$ $14.66 \mathrm{~nm}$, Student's $t$ test $\mathrm{t}_{60}=3.01, P<0.01$ ). Moreover, the average size of GABAergic terminals was decreased after ch'ase (uninjected, $2.19 \pm 0.096 \mu \mathrm{m}^{2}$; ch'ase, $1.79 \pm 0.07 \mu \mathrm{m}^{2}$; Student's $t$ test $\mathrm{t}_{229}=3.34, P=0.001$; Fig. $\left.5 M\right)$, due to an increased percentage of terminals with a small size $\left(\mathrm{X}^{2}{ }_{4}=9.52, P<0.05\right.$; Fig. $5 N)$. The number of release sites per bouton was not different between the uninjected and ch'ase-injected DCN (uninjected, $1.05 \pm 0.069 \mathrm{n} / \mu \mathrm{m}$; ch'ase, $1.17 \pm 0.060 \mathrm{n} / \mu \mathrm{m}$; Student's $t$ test $\mathrm{t}_{147}=1.33, P=0.18$; Fig. $5 O$ ). In addition, no change in the length of release sites was found (uninjected, $300.00 \pm 11.19 \mathrm{~nm}$; ch'ase, $283.68 \pm 9.61 \mathrm{~nm}$; Student's $t$ test $\mathrm{t}_{296}=1.11, P=0.27$; Fig. 5P). Synaptic mitochondria play a crucial role in the maintenance of homeostasis of presynaptic terminals and can divide, fuse, and redistribute within the cell in response to various physiological cues (40). To assess whether ch'ase affects the amount of mitochondria in GABAergic terminals, we evaluated the percentage of area occupied by mitochondria in each terminal. No difference was detected between uninjected and ch'ase-side 

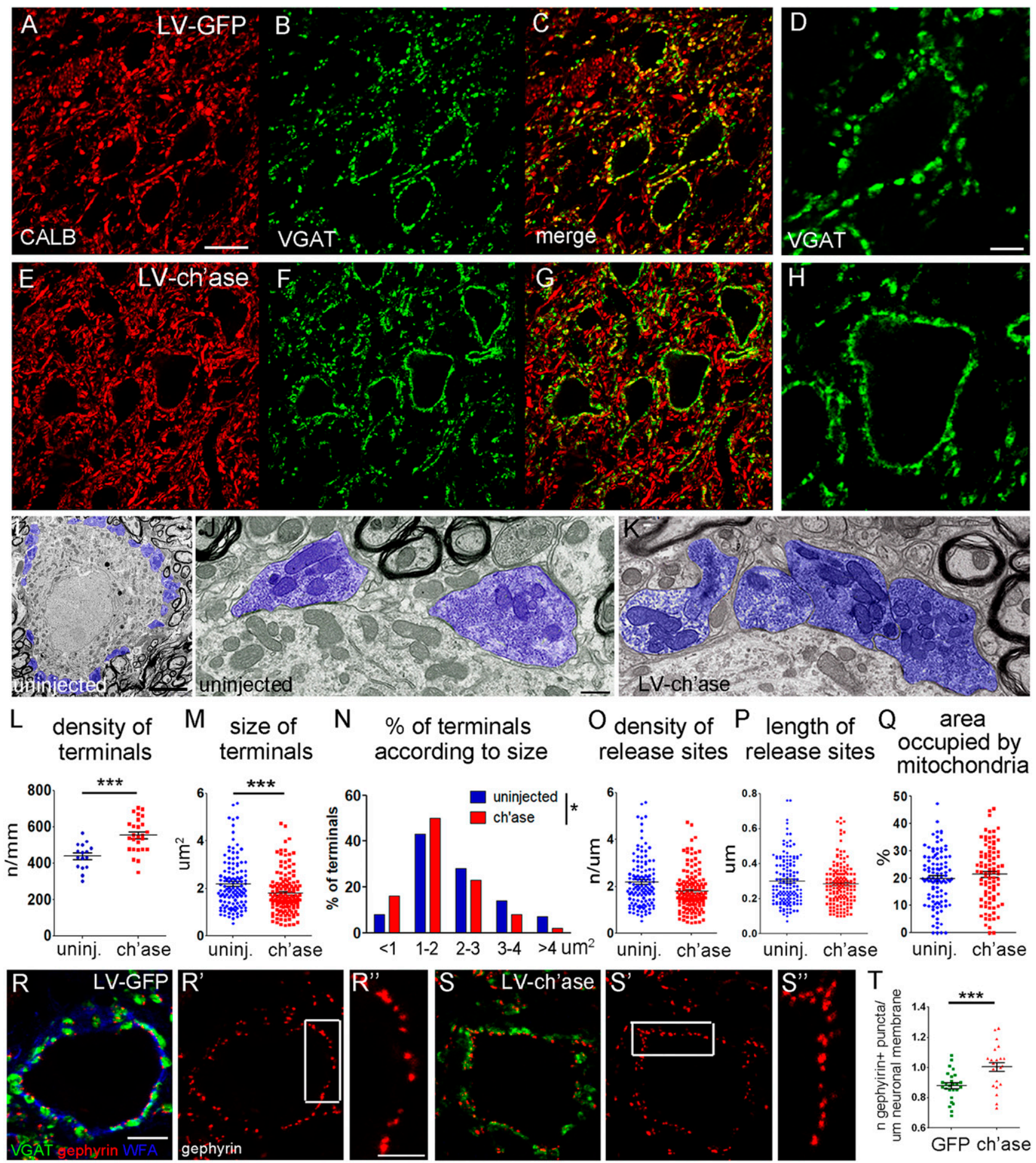

Fig. 5. Effect of ch'ase on plasticity of GABAergic terminals in the DCN. (A-H) Immunolabeling for VGAT and calbindin (CALB) in the IntA of LV-PGK-GFP (A$D)$ and LV-PGK-ch'ase $(E-H)$ injected mice at $4 \mathrm{wk}$ after virus injection. GABAergic terminals, including Purkinje cell boutons, appear as discrete puncta (abundant around DCN neuronal somata) in GFP-injected mice $(A-D)$. See also $S I$ Appendix, Fig. S3. After ch'ase, they show a more continuous distribution ( $E-$ H). See also SI Appendix, Fig. S4. (I) Representative electron micrograph of a control DCN neuron (no LV-ch'ase injection), surrounded by GABAergic terminals (digitally blue color coded). See also SI Appendix, Fig. S5. ( $($ ) GABAergic terminals (blue) in uninjected (uninj.) DCN. (K) GABAergic terminals (blue) in ch'aseinjected DCN. ( $L-Q)$ Quantitations in EM pictures. (L) Density (number per mm neuronal membrane) of GABAergic terminals. Segments of neuronal membrane are shown as individual elements. $(M)$ Size of GABAergic terminals (individual terminals are shown). $(N)$ Frequency distribution of terminals according to size. $(O)$ Number of release sites per $\mu \mathrm{m}$ of membrane of synaptic terminal adjacent to the postsynaptic neuron (individual terminals are shown). $(P)$ Length of individual release sites. $(Q)$ Fraction of the area occupied by mitochondria in individual GABA+ synaptic terminals. $\left(R-S^{\prime \prime}\right)$ Immunolabeling with antigephyrin antibodies (red) shows gephyrin + clusters juxtaposed to GABAergic terminals (which were stained by anti-VGAT antibodies; green) around DCN neurons in LV-GFP $\left(R-R^{\prime \prime}\right)$ and LV-ch'ase mice (S- $\left.S^{\prime \prime}\right)$. WFA (blue) reveals the PNN around a control neuron $(R)$ but is not visible around a ch'ase-treated neuron (S). ( $T$ ) Number of gephyrin+ clusters per $\mu \mathrm{m}$ neuronal membrane (individual membrane segments are shown). *P<0.05, ***P<0.001. (Scale bar, 20 $\mu \mathrm{m}$ in $A$ [also applies to $B, C$, and $E-G$ ], $5 \mu \mathrm{m}$ in $D$ [also applies to $H$ ] and $I, 0.5 \mu \mathrm{m}$ in $J$ [also applies to $K$ ], $5 \mu \mathrm{m}$ in $R$ [also applies to $R^{\prime}, S$, and $S^{\prime}$ ], and $2.5 \mu \mathrm{m}$ in $R^{\prime \prime}$ [also applies to $\left.S^{\prime \prime}\right]$.) 
(uninjected, $19.84 \% \pm 0.98 \%$; ch'ase, $21.33 \% \pm 1.14 \%$; Student's $t$ test $\mathrm{t}_{191}=1.00, P=0.32$; Fig. $\left.5 Q\right)$. Together, these data suggest that ch'ase elicits the formation of new GABAergic terminals in the DCN, which are endowed with release sites and, thus, may be functional.

To further support the hypothesis that newly formed GABAergic presynaptic elements may be functional, we evaluated the number of gephyrin clusters using immunocytochemistry at the light microscopic level. Gephyrin is a scaffold protein that anchors $\mathrm{GABA}_{\mathrm{A}}$ receptors at the postsynaptic membrane of inhibitory synapses and is essential for the formation and stability of the GABAergic synapse (41). We found that the number of gephyrin clusters was higher in ch'ase mice than in control mice (LV-GFP, $0.88 \pm 0.02$ puncta per $\mu \mathrm{m}$ neuronal membrane, $n=26$ neurons; ch'ase, $1.00 \pm 0.03, n=23$ neurons; Student's $t$ test $\mathrm{t}_{47}=3.69, P<0.001$; Fig. $5 R-T$ ), confirming that following PNN digestion, new, functional GABAergic synapses have been formed.

Digestion of PNNs Leads to a Decreased Number of Glutamatergic Terminals. We next evaluated whether ch'ase affects the number and size of glutamatergic terminals. Glutamatergic terminals in the DCN belong to collaterals of mossy fibers and inferior olive axons, which mainly contact the dendritic compartment of DCN neurons (35). Since mossy fiber terminals contain VGLUT1 and/or VGLUT2 and olivary axon terminals contain VGLUT2 $(42,43)$, we visualized glutamatergic terminals by means of anti-VGLUT1 and anti-VGLUT2 antibodies. In control mice, VGLUT1+ and VGLUT2+ puncta were scattered throughout the IntA, with VGLUT1+ terminals being more numerous than VGLUT2+ terminals (Fig. $6 A-C, G$, and $I$ and ref. 43). Four weeks following LV-ch'ase injection, the number of VGLUT1+ terminals was significantly reduced (GFP, 35,301.51 \pm 961.91 terminals per $\mathrm{mm}^{2}$; ch'ase, $30,342.28 \pm 1,011.63$; Student's $t$ test $\mathrm{t}_{12}=3.55, P<0.01$; Fig. $6 A, C, D, F$, and $\left.G\right)$, whereas the number of VGLUT2+ terminals did not significantly change (GFP, $27,969.37 \pm 1,471.89$ terminals per $\mathrm{mm}^{2}$; ch'ase, 29,772.64 \pm 1,591.36; Student's $t$ test $\mathrm{t}_{12}=0.83, P=0.42$; Fig. $6 B, C, E, F$, and I). The size of VGLUT1+ and VGLUT2+ terminals did not change either (VGLUT1, GFP, $1.18 \pm 0.034 \mu \mathrm{m}^{2}$; ch'ase, $1.16 \pm 0.042 \mu \mathrm{m}^{2}$; Student's $t$ test $\mathrm{t}_{12}=0.42, P=0.68$; Fig. $6 H$; VGLUT2, GFP, $1.26 \pm$ $0.019 \mu \mathrm{m}^{2}$; ch'ase, $1.30 \pm 0.011 \mu \mathrm{m}^{2}$; Student's $t$ test $\mathrm{t}_{12}=1.36, P=$ 0.20; Fig. $6 J$ ). Overall, PNN digestion in vivo triggers remarkable plasticity of synaptic connections in the DCN, namely, an increase in the number of inhibitory synapses and a decrease in the number of excitatory terminals.

Effects of ch'ase on DCN Synaptic Terminals During Memory Retention. To examine whether the group difference in retention of the memory trace observed after ch'ase may be due to ch'aseinduced long-term effects on synaptic terminals, we examined GABAergic and glutamatergic terminals in the DLH of mice killed on retention day 21. The DLH is the DCN subnucleus in which mossy fiber sprouting shows a strong positive correlation with EBC rate during learning (27). At the end of EBC retention phase, GABAergic terminals around neuronal cell bodies in ch'ase-treated mice were less discrete than in GFP mice, as revealed following VGAT staining. Indeed, we detected a significantly lower number of intersynaptic spaces (GFP, $170.11 \pm 5.50$ troughs in VGAT intensity profile per mm neuronal membrane, $n=56$ neurons out of 4 mice; ch'ase: $108.43 \pm 5.48, n=77$ neurons out of 5 mice; Student's $t$ test $\mathrm{t}_{131}=7.75, P<0.001$; SI Appendix, Fig. S6 $\left.A-C\right)$. As to glutamatergic terminals, we found a significant decrease in VGLUT1 density in ch'ase-treated mice when compared to GFP mice (GFP, 46,221.63 \pm 625.74; ch'ase, 41,387.06 $\pm 1,516.97$; Student's $t$ test $\mathrm{t}_{6}=$ 2.95, $P<0.05$; SI Appendix, Fig. S6 $D-F)$. These data demonstrate that ch'ase induces long-term synaptic changes, which may underlie the observed decline in memory retention.
PNN Digestion Alters Electrophysiological Properties of DCN Neurons in Awake Mice. To evaluate the role of PNNs in the regulation of spike activity of DCN neurons in vivo, we made extracellular single unit recordings targeted at the lateral IntA and DLH in awake behaving mice. Recordings were obtained from LV-GFP ( $n=35$ neurons in 6 mice) and LV-ch'ase mice $(n=38$ neurons in 5 mice). Recording location was estimated post hoc after visualization of neurobiotin, which was added to the pipette solution and pressure-injected at the recording site. Neurobiotin staining appeared most prominent in the IntA (SI Appendix, Fig. S7 $A-C$ ), confirming that our recordings were targeted to the correct region. Furthermore, we only included neurons that had been recorded in the IntA DLH region with fully digested PNNs, as evaluated by post hoc WFA staining. Spontaneous spike activity was recorded in awake mice. For both groups we observed a wide range of basal firing frequencies (Fig. 7), possibly representing different neuronal types with varying physiological properties $(37,44,45)$. Interestingly, we found that the average baseline firing frequency of recorded neurons in LV-ch'ase mice was significantly lower than that in LV-GFP mice (LV-GFP, $53.8 \pm 5.39 \mathrm{~Hz}$; LV-ch'ase, $35.6 \pm 5.08 \mathrm{~Hz}$; Mann-Whitney $U$ test $\mathrm{U}=465, P=0.027$; Fig. $7 A$ and $B$ ). No difference in coefficient of variation (CV) of spiking (LV-GFP, $0.58 \pm 0.04$; LVch'ase, $0.80 \pm 0.10$; MWU test $\mathrm{U}=730, P=0.343$; Fig. $7 C$ ) or average $\mathrm{CV}$ of two adjacent interspike intervals $\left(\mathrm{CV}_{2} ; \mathrm{LV}-\mathrm{GFP}\right.$, $0.50 \pm 0.02$; LV-ch'ase, $0.54 \pm 0.03$; Student's $t$ test $\mathrm{t}_{64.86}=$ $-0.821, P=0.415$; Fig. $7 D$ ) was found between groups. These findings indicate that ch'ase-induced digestion of PNNs in the IntA leads to lower neuronal baseline spike activity in vivo, without obviously affecting the regularity of firing.

\section{Discussion}

In this study we have demonstrated that 1) PNNs in the DCN are reduced during $\mathrm{EBC}$ memory acquisition but are restored when memories are consolidated; 2) digestion of PNNs in the DCN increases and accelerates EBC learning rate but impairs memory retention; 3) PNN digestion causes substantial remodeling of DCN connections, with an increase in inhibitory synapses and a decrease in excitatory synapses; and 4) PNN digestion induces a reduction in the spontaneous firing activity of DCN neurons in vivo. Overall, we show that PNN modulation is a critical factor for dynamic control of DCN connectivity and, consequently, cerebellum-dependent learning.

PNN Dynamics and EBC. We found that PNNs exhibit very dynamic changes during acquisition and consolidation of cerebellumdependent associative memories. Acquisition of EBC memories was accompanied by a decrease in the expression of PNN chondroitin sulfates in DCN areas that are involved in the control of this type of learning, whereas, after consolidation of EBC memories, PNN chondroitin sulfate levels were not different from the control situation. PNN modulation was not limited to CSPG-sugar chains, since the CSPG aggrecan, one of the crucial proteins for PNN assembly and stability (6), was also decreased during learning. CSPGs are known to inhibit axonal growth (46). Moreover, molecules interacting with CSPGs in the nets, such as Semaphorin3A (Sema3A) and Otx2, inhibit plasticity $(7,18)$. Sema3A is also present in the DCN, and Sema3A downregulation is associated with axonal remodeling therein (47). Thus, we propose that reduced levels of PNN-CSPGs during EBC acquisition may allow synaptic remodeling, which, in turn, may drive the learning process. On the other hand, restored expression levels of CSPGs might promote synapse stabilization (48), which is necessary for the subsequent maintenance of memory traces (49).

Expression of PNNs in the brain, including the DCN, has been previously shown to be reduced following enriched environmental stimulation $(32,50)$, locomotion training $(51)$, injury $(47$, 

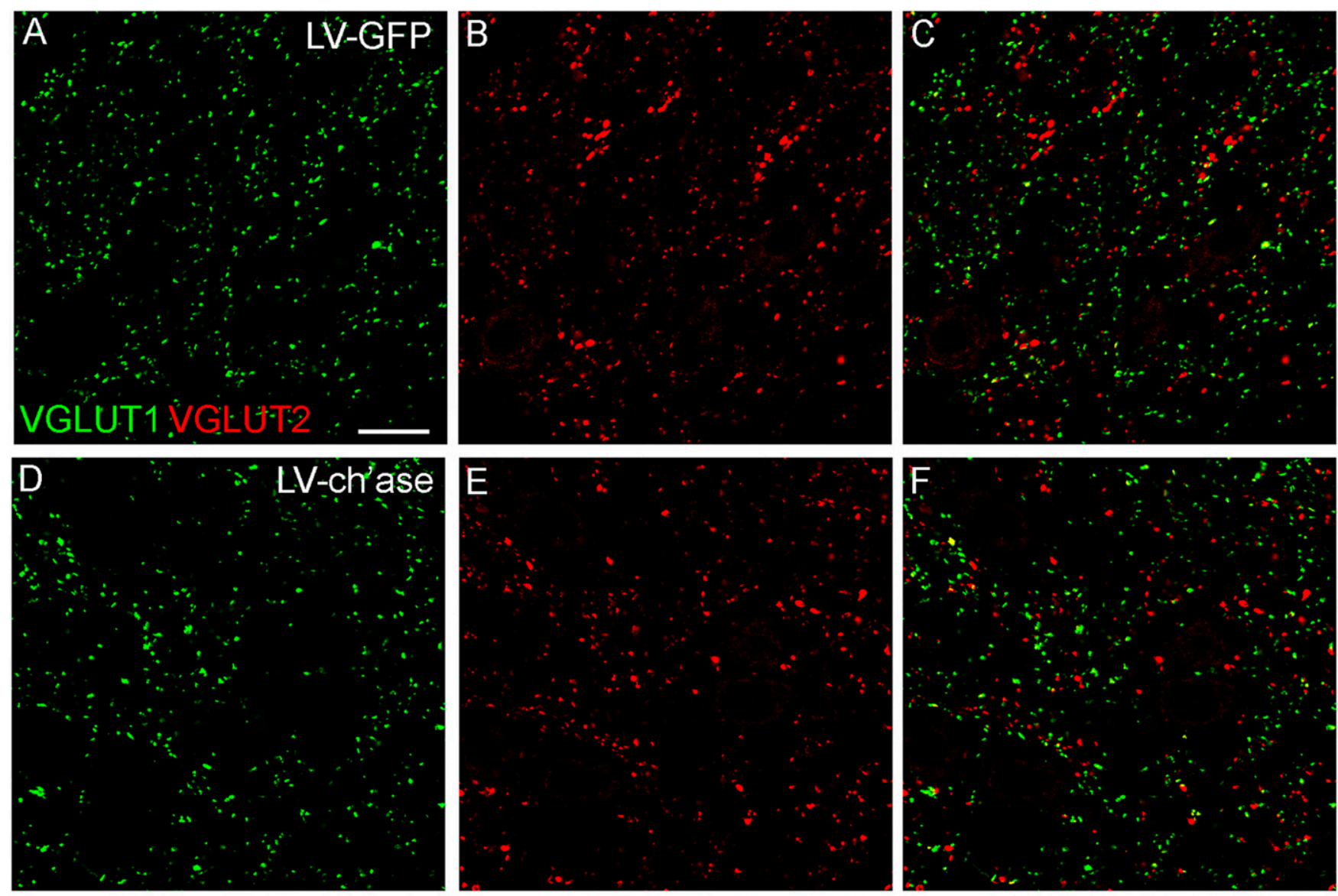

G

$\mathrm{H}$

VGLUT1 density

VGLUT1 size

I

VGLUT2 density

$\mathrm{J}$
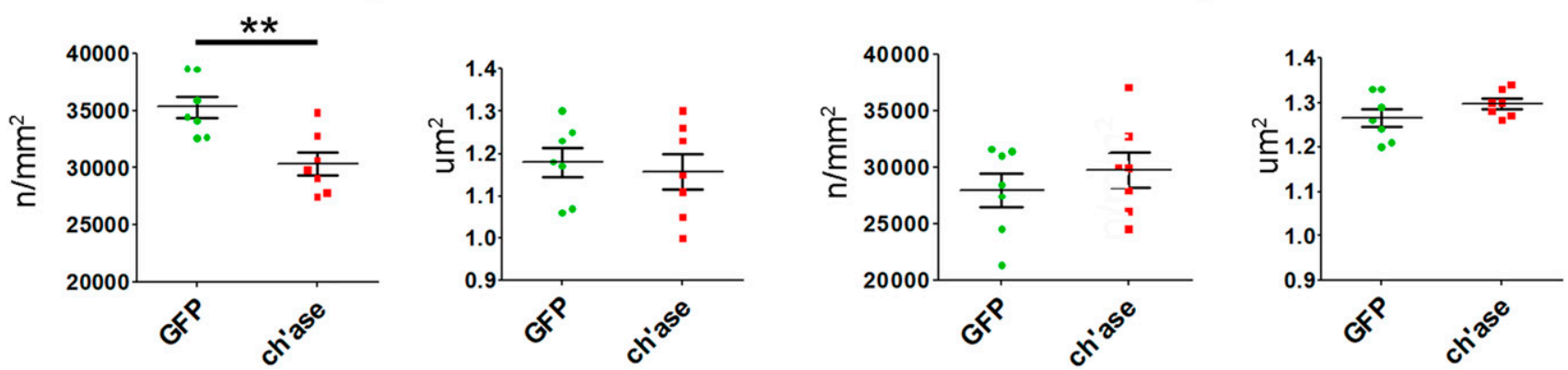

Fig. 6. Effect of ch'ase on plasticity of glutamatergic terminals in the DCN. (A-F) Immunolabeling for VGLUT1 (green) and VGLUT2 (red) in the IntA of LVPGK-GFP $(A-C)$ and LV-PGK-ch'ase $(D-F)$ injected mice at 4 wk after virus injection. The number of VGLUT1+ terminals per mm ${ }^{2}$ (density) is significantly reduced after ch'ase $(G)$, whereas VGLUT2 density remains unchanged $(I)$. See also $S /$ Appendix, Fig. S6. The size of VGLUT1+ $(H)$ and VGLUT2+ terminals $(J)$ is not different between control and ch'ase mice. ${ }^{* *} P<0.01$. (Scale bar, $25 \mu \mathrm{m}$ in $A$ [also applies to $B-F$ ].)

52, 53), or drug exposure (54-56). Our study shows a change in PNN expression during learning of new associations. In the context of learning and memory, dynamic regulation of PNNs around Golgi neurons in the cerebellar cortex has also been shown to be an important component of drug memory retention (15). Moreover, PNNs in the auditory cortex are increased after fear conditioning (14). Thus, while elaborating on the work of others, our study indicates that the expression of PNNs can be up- and down-regulated during the acquisition and consolidation of newly formed associations, respectively.

Decreased expression of PNN components can occur by cleaving them with proteases, such as matrix metalloproteinases (MMPs) and ADAMTSs (A Disintegrin and Metalloprotease with thrombospondin motifs) (57-59). In the cerebellum, MMP-9 mediates plasticity events induced by enriched environmental stimulation and contributes to a decrease in PNNs (60). Therefore, EBC may induce a finely tuned, time-dependent modulation of such enzymes which, in turn, may control PNN stability. Moreover, because enriched environment as well as fear conditioning induce changes in the mRNA levels for crucial PNN components, such as aggrecan, link proteins, and hyaluronan $(14,32)$, EBC may well exert a similar effect on the synthetic machinery of PNN molecules.

PNN Digestion and Behavioral Impact. Our observations of a dynamic regulation of PNN-CSPGs during EBC prompted us to examine whether PNN changes play a causative role in EBC learning and 

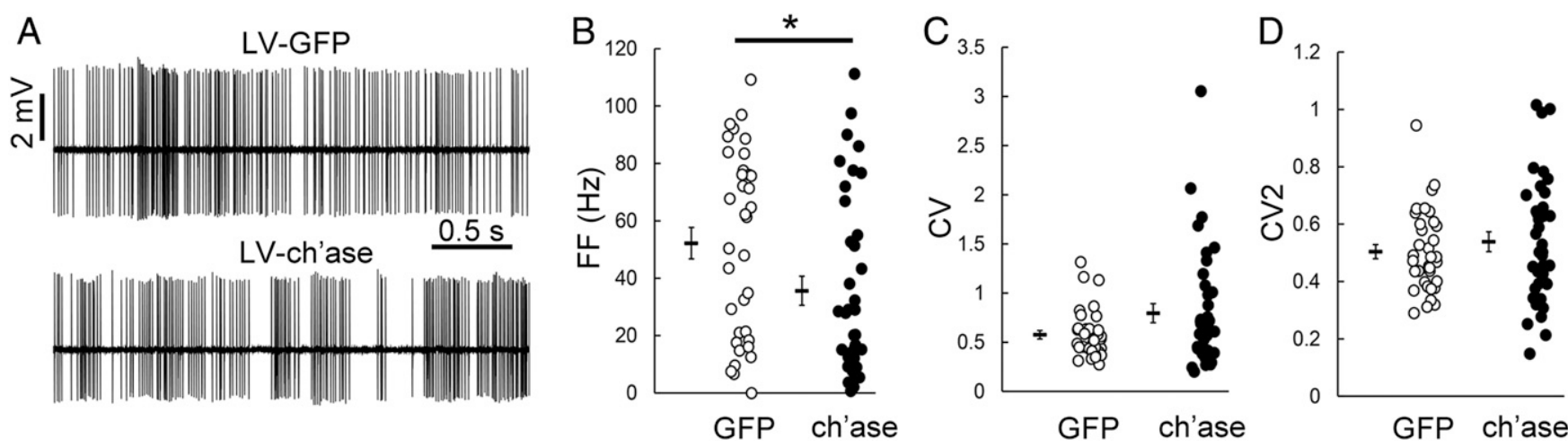

Fig. 7. Spontaneous activity of IntA neurons after PNN digestion is reduced. (A) Representative traces of extracellular neuronal recordings in awake behaving LV-GFP (Upper) and LV-ch'ase mice (Lower). The firing frequency (FF) of IntA neurons is significantly reduced in LV-ch'ase mice when compared to LVGFP mice $(B)$, whereas $C V(C)$ and $C_{2}$ values $(D)$ are comparable. See $S I$ Appendix, Fig. S7, for recording locations. ${ }^{*}<0.05$.

memory. Therefore, we enzymatically digested PNN-CSPGs in the DCN by overexpressing ch'ase in a lentiviral vector. The ch'ase gene, which is expressed in its original format in bacteria, was modified to allow efficient secretion of active chondroitinase from mammalian cells (61). The viral approach ensures long-term expression of the enzyme (62). In the cerebellum, we observed strong PNN-CSPG digestion in the DCN, which resulted in substantial acceleration and amelioration of learning. Direct injection of the enzyme into the DCN has a smaller effect, inducing partial digestion of CSPGs and an increase in the learning rate only at late stages of the acquisition phase (36). Thus, removal of PNNCSPGs with our lentiviral approach induced faster and better associative motor learning, allowing us to establish a causal role for a decrease in PNN during acquisition. The impact of PNN digestion on learning has also been studied in the context of cognitive or emotional processes. For example, digestion of PNNs in the auditory cortex results in enhanced performance in animals trained in a cue reversal learning task (8). Likewise, degradation of PNNs in the amygdala can induce erasure of fear memories or drug memories via extinction $(12,63)$, which is a form of learning. Importantly, our study shows that digestion of PNN-CSPGs causes a decrease in EBC retention over time, suggesting that PNNs are implicated in the consolidation of previously acquired associative motor memories. Although PNN digestion in cortical areas negatively affects the storage of drug-associated memories (13) and fear memories (14, 16), it has opposite effects on object recognition memory (11). This suggests that PNNs may play different roles in distinct types of memory (e.g., working memories versus associative memories), depending on the organization and timing-dependent properties of the underlying neuronal network involved.

It is interesting to speculate on a role of DCN PNNs in the control of a critical period for retention of associative motor memories regulated by the cerebellum. Retention of EBC memories in rats is labile when memories are acquired at young age (postnatal day 17) but not when they are acquired in adulthood $(64,65)$. Notably, PNNs in the DCN are still very immature between P14 and P21 (66), raising the possibility that memory consolidation in the DCN is subject to a critical period during early postnatal development, which depends on PNN maturation.

PNNs and DCN Synaptic Connectivity. Our study shows that synaptic connectivity in the DCN is profoundly altered when PNN-CSPGs are digested as we observed a shift of the excitatory/inhibitory balance toward an increased inhibition. Ch'ase induced a strong increase in the number of Purkinje cell synapses on DCN neurons. Purkinje cells are essential for the control of EBC acquisition rate and timing (67). Therefore, fine-regulating Purkinje cell input may facilitate learning. In contrast to our results, the study by Hirono et al. (36) shows no structural modifications of Purkinje cell terminals in acute cerebellar slices treated with ch'ase. This discrepancy could be explained by a different efficacy of ch'ase (25\% PNN reduction in ref. 36 versus $90 \%$ PNN reduction in our study), different age of the animals (adolescent versus adult), and/or use of different models (in vitro versus in vivo). Overall, our data strongly support a role for PNN-CSPGs in restricting Purkinje axon growth and synapse formation. Likewise, the growth potential of Purkinje axon collaterals in the cerebellar cortex is under control of CSPGs that are diffusely distributed in the neuropil (68).

We also found that ch'ase induced a decrease in the density of VGLUT1+ terminals in the DLH and adjacent areas of the IntA. The observed differential effects of ch'ase on distinct types of cerebellar synapses might be due to a specific distribution of receptors for CSPGs (69) and/or for Sema3A (19). Purkinje cells show a strong expression of receptors for CSPG-chondroitin sulfates, such as PTPsigma and NgR3 (Allen brain atlas), suggesting that Purkinje axon terminals may be directly affected by chondroitin sulfate digestion.

Pontine mossy fibers, which relay the CS to the cerebellum, express VGLUT1+ (Allen Brain Atlas). The pontine mossy fibers in the DLH sprout during EBC but not during pseudoconditioning with the same set of sensory inputs, and the number of sprouting fibers can be correlated to the amplitude of the CR (27). We hypothesize that in ch'ase-treated mice, mossy fiber synaptic contacts formed during the acquisition phase may, due to the absence of PNNs, not be stabilized, and as a consequence, the memory trace cannot be consolidated. Thus, a net increase in the inhibitory tone onto DCN neurons may promote initially an enhanced learning rate, whereas a higher level of baseline excitation may be important for memory retention. How can this be reconciled with our observation that PNNs are also important for the baseline electrophysiological properties of DCN neurons? After ch'ase treatment, DCN neurons of awake behaving mice showed lower spontaneous firing frequencies. This change in the baseline firing rate of $\mathrm{DCN}$ neurons may be partly explained by the increased Purkinje cell innervation and/or the decreased density of excitatory terminals. Nonetheless, due to a presumed role of PNNs in preventing the free diffusion of potassium or sodium ions in the extracellular space (70), we cannot exclude that a decreased reservoir of available cations following ch'ase administration also provides a more direct impact on the firing frequency of DCN neurons. Importantly, reduced baseline activity of DCN neurons may affect the level of rebound firing (71, 72 ), which in turn may play a role in the induction of DCN plasticity and the strength of the CR $(73,74)$. Moreover, since the simple spike activity of Purkinje cells is temporarily suppressed during the actual expression of a CR (75-77), we expect the level of rebound firing in 
the DCN during $\mathrm{CR}$ expression to be reduced when their baseline firing is lower (26).

Conclusions. We provide compelling evidence that PNNs are indispensable for the control of specific connection patterns in the DCN and for regulating functional properties of DCN neurons. Patterns of not only modulatory but also baseline activity of DCN neurons are essential for the formation and expression of associative memories.

Since the cerebellum controls not only motor functions but also social, cognitive, and emotional functions $(22,78-80)$, it will be interesting to determine whether PNNs are also implicated in other cerebellum-dependent processes, including both physiological and pathological conditions.

\section{Materials and Methods}

Adult male C57BL/6J mice (6 to 8 wk old; Janvier Laboratories) were socially housed with food and water ad libitum, in 12-h light and dark cycles. All procedures were approved by the animal committee of the Royal Dutch Academy of Arts and Sciences and adhered to the European guidelines for the care and use of laboratory animals (Council Directive 86/6009/EEC).

1. J. W. Fawcett, T. Oohashi, T. Pizzorusso, The roles of perineuronal nets and the perinodal extracellular matrix in neuronal function. Nat. Rev. Neurosci. 20, 451-465 (2019).

2. T. Pizzorusso et al., Reactivation of ocular dominance plasticity in the adult visual cortex. Science 298, 1248-1251 (2002).

3. D. Carulli et al., Animals lacking link protein have attenuated perineuronal nets and persistent plasticity. Brain 133, 2331-2347 (2010).

4. C. Bernard, A. Prochiantz, Otx2-PNN interaction to regulate cortical plasticity. Neural Plast. 2016, 7931693 (2016).

5. H. H. C. Lee et al., Genetic Otx2 mis-localization delays critical period plasticity across brain regions. Mol. Psychiatry 22, 680-688 (2017).

6. D. Rowlands et al., Aggrecan directs extracellular matrix-mediated neuronal plasticity. J. Neurosci. 38, 10102-10113 (2018)

7. E. M. Boggio et al., Inhibition of Semaphorin3A promotes ocular dominance plasticity in the adult rat visual cortex. Mol. Neurobiol. 56, 5987-5997 (2019).

8. M. F. K. Happel et al., Enhanced cognitive flexibility in reversal learning induced by removal of the extracellular matrix in auditory cortex. Proc. Natl. Acad. Sci. U.S.A. 111 2800-2805 (2014).

9. R. Frischknecht et al., Brain extracellular matrix affects AMPA receptor lateral mobility and short-term synaptic plasticity. Nat. Neurosci. 12, 897-904 (2009).

10. J. M. Massey et al., Chondroitinase $A B C$ digestion of the perineuronal net promotes functional collateral sprouting in the cuneate nucleus after cervical spinal cord injury. J. Neurosci. 26, 4406-4414 (2006)

11. C. Romberg et al., Depletion of perineuronal nets enhances recognition memory and long-term depression in the perirhinal cortex. J. Neurosci. 33, 7057-7065 (2013).

12. Y.-X. Xue et al., Depletion of perineuronal nets in the amygdala to enhance the erasure of drug memories. J. Neurosci. 34, 6647-6658 (2014).

13. M. Slaker et al., Removal of perineuronal nets in the medial prefrontal cortex impairs the acquisition and reconsolidation of a cocaine-induced conditioned place preference memory. J. Neurosci. 35, 4190-4202 (2015).

14. S. B. Banerjee et al., Perineuronal nets in the adult sensory cortex are necessary for fear learning. Neuron 95, 169-179.e3 (2017).

15. M. Carbo-Gas et al., Cerebellar perineuronal nets in cocaine-induced pavlovian memory: Site matters. Neuropharmacology 125, 166-180 (2017).

16. E. H. Thompson et al., Removal of perineuronal nets disrupts recall of a remote fear memory. Proc. Natl. Acad. Sci. U.S.A. 115, 607-612 (2018).

17. J. M. Blacktop, B. A. Sorg, Perineuronal nets in the lateral hypothalamus area regulate cue-induced reinstatement of cocaine-seeking behavior. Neuropsychopharmacology 44, 850-858 (2019).

18. M. Beurdeley et al., Otx2 binding to perineuronal nets persistently regulates plasticity in the mature visual cortex. J. Neurosci. 32, 9429-9437 (2012).

19. T. Vo et al., The chemorepulsive axon guidance protein semaphorin $3 \mathrm{~A}$ is a constituent of perineuronal nets in the adult rodent brain. Mol. Cell. Neurosci. 56, 186-200 (2013).

20. G. Dick et al., Semaphorin $3 A$ binds to the perineuronal nets via chondroitin sulfate type E motifs in rodent brains. J. Biol. Chem. 288, 27384-27395 (2013).

21. D. Carulli et al., Composition of perineuronal nets in the adult rat cerebellum and the cellular origin of their components. J. Comp. Neurol. 494, 559-577 (2006).

22. D. Timmann et al., The human cerebellum contributes to motor, emotional and cognitive associative learning. A review. Cortex 46, 845-857 (2010).

23. J. H. Freeman, A. B. Steinmetz, Neural circuitry and plasticity mechanisms underlying delay eyeblink conditioning. Learn. Mem. 18, 666-677 (2011).

24. C. I. De Zeeuw, C. H. Yeo, Time and tide in cerebellar memory formation. Curr. Opin. Neurobiol. 15, 667-674 (2005).

25. J. R. Pugh, I. M. Raman, Nothing can be coincidence: Synaptic inhibition and plasticity in the cerebellar nuclei. Trends Neurosci. 32, 170-177 (2009).

26. M. M. Ten Brinke et al., Dynamic modulation of activity in cerebellar nuclei neurons during pavlovian eyeblink conditioning in mice. elife 6, 1-27 (2017).
A detailed description of experimental procedures and analyses is provided in SI Appendix, SI Materials and Methods.

Data Deposition. Datasets and analyses have been deposited in the online open access repository Figshare and can be accessed via https://figshare.com/ collections/Cerebellar_plasticity_and_associative_memories_are_controlled_ by_perineuronal_nets/4814616/1.

ACKNOWLEDGMENTS. The work was supported by University of Turin, La Maratò de TV3, International Foundation for Research in Paraplegia, Inter national Spinal Research Trust, the Netherlands Institute for Neuroscience, the Netherlands Organization for Scientific Research (Earth and Life Sciences), the Dutch Organization for Medical Sciences European Research Council-advanced, and European Research Council- Proof of Concept. We are grateful to Bas Koekkoek, Ilja IJpelaar, and Michiel ten Brinke (Erasmus Medical Center, Rotterdam, The Netherlands) for their help with the eyeblink conditioning paradigm, Willemijn Ranzijn (Netherlands Institute for Neuroscience, Amsterdam, The Netherlands) for her help with behavioral experiments and immunohistochemistry; and Barbara Hobo, Cynthia Geelen, and Anna Court (Netherlands Institute for Neuroscience, Amsterdam, The Netherlands) for their excellent technical support.

27. H. J. Boele, S. K. E. Koekkoek, C. I. De Zeeuw, T. J. H. Ruigrok, Axonal sprouting and formation of terminals in the adult cerebellum during associative motor learning. $J$. Neurosci. 33, 17897-17907 (2013)

28. S. A. Heiney, M. P. Wohl, S. N. Chettih, L. I. Ruffolo, J. F. Medina, Cerebellardependent expression of motor learning during eyeblink conditioning in headfixed mice. J. Neurosci. 34, 14845-14853 (2014).

29. A. Rasmussen, A. C. H. G. Ijpelaar, C. I. De Zeeuw, H. J. Boele, Caffeine has no effect on eyeblink conditioning in mice. Behav. Brain Res. 337, 252-255 (2018).

30. W. Härtig, K. Brauer, G. Brückner, Wisteria floribunda agglutinin-labelled nets surround parvalbumin-containing neurons. Neuroreport 3, 869-872 (1992).

31. D. J. Krupa, R. F. Thompson, Reversible inactivation of the cerebellar interpositus nucleus completely prevents acquisition of the classically conditioned eye-blink response. Learn. Mem. 3, 545-556 (1997).

32. S. Foscarin et al., Experience-dependent plasticity and modulation of growth regulatory molecules at central synapses. PLoS One 6, e16666 (2011).

33. M. Ivarsson, G. Hesslow, Bilateral control of the orbicularis oculi muscle by one cerebellar hemisphere in the ferret. Neuroreport 4, 1127-1130 (1993).

34. K. D. Bosch, E. J. Bradbury, J. Verhaagen, J. W. Fawcett, S. B. McMahon, Chondroitinase $A B C$ promotes plasticity of spinal reflexes following peripheral nerve injury. Exp. Neurol. 238, 64-78 (2012).

35. C. I. De Zeeuw, A. S. Berrebi, Postsynaptic targets of Purkinje cell terminals in the cerebellar and vestibular nuclei of the rat. Eur. J. Neurosci. 7, 2322-2333 (1995).

36. M. Hirono et al., Perineuronal nets in the deep cerebellar nuclei regulate GABAergic transmission and delay eyeblink conditioning. J. Neurosci. 38, 6130-6144 (2018)

37. M. Uusisaari, K. Obata, T. Knöpfel, Morphological and electrophysiological properties of GABAergic and non-GABAergic cells in the deep cerebellar nuclei. J. Neurophysiol. 97, 901-911 (2007)

38. K. Uchizono, Characteristics of excitatory and inhibitory synapses in the central nervous system of the cat. Nature 207, 642-643 (1965).

39. J. A. Kleim et al., Synapse formation is associated with memory storage in the cerebellum. Proc. Natl. Acad. Sci. U.S.A. 99, 13228-13231 (2002).

40. M. Vos, E. Lauwers, P. Verstreken, Synaptic mitochondria in synaptic transmission and organization of vesicle pools in health and disease. Front. Synaptic Neurosci. 2, 139 (2010).

41. S. K. Tyagarajan, J. M. Fritschy, Gephyrin: A master regulator of neuronal function? Nat. Rev. Neurosci. 15, 141-156 (2014).

42. H. Hioki et al., Differential distribution of vesicular glutamate transporters in the rat cerebellar cortex. Neuroscience 117, 1-6 (2003).

43. H. Mao, S. Hamodeh, F. Sultan, Quantitative comparison of vesicular glutamate transporters in rat deep cerebellar nuclei. Neuroscience 376, 152-161 (2018).

44. C. B. Canto, L. Witter, C. I. De Zeeuw, Whole-cell properties of cerebellar nuclei neurons in vivo. PLoS One 11, e0165887 (2016).

45. O. Orkan Özcan et al., Differential coding strategies in glutamatergic and gabaergic neurons in the medial cerebellar nucleus. J. Neurosci. 40, 159-170 (2019).

46. J. C. F. Kwok, F. Afshari, G. García-Alías, J. W. Fawcett, Proteoglycans in the central nervous system: Plasticity, regeneration and their stimulation with chondroitinase ABC. Restor. Neurol. Neurosci. 26, 131-145 (2008).

47. D. Carulli, S. Foscarin, A. Faralli, E. Pajaj, F. Rossi, Modulation of semaphorin3A in perineuronal nets during structural plasticity in the adult cerebellum. Mol. Cell. Neurosci. 57, 10-22 (2013).

48. M. Geissler et al., Primary hippocampal neurons, which lack four crucial extracellular matrix molecules, display abnormalities of synaptic structure and function and severe deficits in perineuronal net formation. J. Neurosci. 33, 7742-7755 (2013).

49. E. R. Kandel, Y. Dudai, M. R. Mayford, The molecular and systems biology of memory. Cell 157, 163-186 (2014)

50. A. Sale et al., Environmental enrichment in adulthood promotes amblyopia recovery through a reduction of intracortical inhibition. Nat. Neurosci. 10, 679-681 (2007). 
51. C. C. Smith et al., Differential regulation of perineuronal nets in the brain and spina cord with exercise training. Brain Res. Bull. 111, 20-26 (2015).

52. S. T. Carmichael et al., Growth-associated gene expression after stroke: Evidence for a growth-promoting region in peri-infarct cortex. Exp. Neurol. 193, 291-311 (2005).

53. A. Faralli et al., Modifications of perineuronal nets and remodelling of excitatory and inhibitory afferents during vestibular compensation in the adult mouse. Brain Struct. Funct. 221, 3193-3209 (2016).

54. M. C. Van den Oever et al., Extracellular matrix plasticity and GABAergic inhibition of prefrontal cortex pyramidal cells facilitates relapse to heroin seeking. Neuropsychopharmacology 35, 2120-2133 (2010).

55. D. Vazquez-Sanroman et al., The cerebellum on cocaine: Plasticity and metaplasticity. Addict. Biol. 20, 941-955 (2015).

56. M. L. Slaker et al., Cocaine exposure modulates perineuronal nets and synaptic ex citability of fast-spiking interneurons in the medial prefrontal cortex. eNeuro ENEURO.0221-18.2018 (2018).

57. M. D. Tortorella et al., Purification and cloning of aggrecanase-1: A member of the ADAMTS family of proteins. Science 284, 1664-1666 (1999).

58. H. Nakamura et al., Brevican is degraded by matrix metalloproteinases and aggrecanase-1 (ADAMTS4) at different sites. J. Biol. Chem. 275, 38885-38890 (2000).

59. C. Levy, J. M. Brooks, J. Chen, J. Su, M. A. Fox, Cell-specific and developmental expression of lectican-cleaving proteases in mouse hippocampus and neocortex. $J$. Comp. Neurol. 523, 629-648 (2015).

60. V. Stamenkovic et al., The extracellular matrix glycoprotein tenascin-C and matrix metalloproteinases modify cerebellar structural plasticity by exposure to an enriched environment. Brain Struct. Funct. 222, 393-415 (2017).

61. E. M. Muir et al., Modification of N-glycosylation sites allows secretion of bacterial chondroitinase ABC from mammalian cells. J. Biotechnol. 145, 103-110 (2010).

62. R. R. Zhao et al., Lentiviral vectors express chondroitinase $A B C$ in cortical projections and promote sprouting of injured corticospinal axons. J. Neurosci. Methods 201, 228238 (2011).

63. N. Gogolla, P. Caroni, A. Lüthi, C. Herry, Perineuronal nets protect fear memories from erasure. Science 325, 1258-1261 (2009).

64. D. A. Nicholson, J. A. Sweet, J. H. Freeman, Jr, Long-term retention of the classically conditioned eyeblink response in rats. Behav. Neurosci. 117, 871-875 (2003).

65. K. L. Brown, J. H. Freeman, Retention of eyeblink conditioning in periweanling and adult rats. Dev. Psychobiol. 58, 1055-1065 (2016).
66. D. Carulli, K. E. Rhodes, J. W. Fawcett, Upregulation of aggrecan, link protein 1, and hyaluronan synthases during formation of perineuronal nets in the rat cerebellum. $J$. Comp. Neurol. 501, 83-94 (2007).

67. K. M. Christian, R. F. Thompson, Neural substrates of eyeblink conditioning: Acquisition and retention. Learn. Mem. 10, 427-455 (2003).

68. L. Corvetti, F. Rossi, Degradation of chondroitin sulfate proteoglycans induces sprouting of intact purkinje axons in the cerebellum of the adult rat. J. Neurosci. 25, 7150-7158 (2005).

69. J. A. Duncan, R. Foster, J. C. F. Kwok, The potential of memory enhancement through modulation of perineuronal nets. Br. J. Pharmacol. 176, 3611-3621 (2019).

70. W. Härtig et al., Cortical neurons immunoreactive for the potassium channel Kv3.1b subunit are predominantly surrounded by perineuronal nets presumed as a buffering system for cations. Brain Res. 842, 15-29 (1999).

71. C. D. Aizenman, D. J. Linden, Regulation of the rebound depolarization and spontaneous firing patterns of deep nuclear neurons in slices of rat cerebellum. J. Neurophysiol. 82, 1697-1709 (1999).

72. N. Zheng, I. M. Raman, Ca currents activated by spontaneous firing and synaptic disinhibition in neurons of the cerebellar nuclei. J. Neurosci. 29, 9826-9838 (2009).

73. D. Z. Wetmore, E. A. Mukamel, M. J. Schnitzer, Lock-and-key mechanisms of cerebellar memory recall based on rebound currents. J. Neurophysiol. 100, 2328-2347 (2008).

74. C. I. De Zeeuw et al., Spatiotemporal firing patterns in the cerebellum. Nat. Rev. Neurosci. 12, 327-344 (2011).

75. A. Rasmussen, D. A. Jirenhed, G. Hesslow, Simple and complex spike firing patterns in Purkinje cells during classical conditioning. Cerebellum 7, 563-566 (2008).

76. H. E. Halverson, A. Khilkevich, M. D. Mauk, Relating cerebellar purkinje cell activity to the timing and amplitude of conditioned eyelid responses. J. Neurosci. 35, 7813-7832 (2015).

77. M. M. ten Brinke et al., Evolving models of pavlovian conditioning: Cerebellar cortical dynamics in awake behaving mice. Cell Rep. 13, 1977-1988 (2015).

78. B. Sacchetti, E. Baldi, C. A. Lorenzini, C. Bucherelli, Cerebellar role in fear-conditioning consolidation. Proc. Natl. Acad. Sci. U.S.A. 99, 8406-8411 (2002)

79. Z. Gao et al., A cortico-cerebellar loop for motor planning. Nature 563, 113-116 (2018).

80. S. S. H. Wang, A. D. Kloth, A. Badura, The cerebellum, sensitive periods, and autism. Neuron 83, 518-532 (2014).

81. K. Franklin, G. Paxinos, The Mouse Brain in Stereotaxic Coordinates (Academic Press, 2001). 